\title{
DERECHO AL EMPLEO DE LAS PERSONAS CON DISCAPACIDAD: DE OBJETOS DE PROTECCIÓN SOCIAL A SUJETOS DE DERECHOS
}

\author{
Autora: Virginia Torre \\ viritorre@hotmail.com \\ Universidad Nacional de Córdoba (Argentina) \\ y Fundación Gaude (Córdoba, Argentina)
}

\begin{abstract}
Resumen
Las personas con discapacidad sufren a lo largo de sus vidas de discriminación que les lleva a estar limitados en el ejercicio pleno de sus derechos. El cambio que se ha producido desde la aprobación de la Convención sobre los Derechos de las Personas con Discapacidad (CPCD, por sus siglas en inglés) ha generado la necesidad de que los Estados adapten sus normas mediante el establecimiento de acciones de inclusión concretas. Hoy en día, un alto porcentaje de la población mundial tiene una discapacidad, lo que denota la importancia de este debate para la sociedad Internacional. Este artículo tiene el objetivo de ofrecer una visión general de los aspectos que hacen al reconocimiento de las personas con discapacidad como sujetos de derechos, específicamente en las oportunidades en el acceso a un empleo. El propósito es dar una perspectiva
\end{abstract}


sobre las barreras reales que enfrentan este grupo de personas cuando participan plenamente en el desarrollo de sus vidas. Ofreciendo una perspectiva multidisciplinaria, destacando el trabajo conjunto con organizaciones no gubernamentales, específicamente profesionales en el área, mediante acciones concretas para erradicar políticas discriminatorias y generar espacios para la inclusión y la igualdad desde una edad temprana.

Palabras claves: discapacidad; Derechos Humanos; inclusión; acceso al empleo.

\title{
Right to employment of persons with disabilities: from being objects of social protection to be holders of rights
}

\begin{abstract}
Persons with disabilities suffer during their lives discrimination that leads them to be limited in the full exercise of their rights. The change that has taken place since the adoption of the Convention on the Rights of Persons with Disabilities (CPCD) has generated the obligation of the States to adapt their standards through the establishment of concrete inclusion actions. Nowadays, a high percentage of the world population has a disability, which denotes the importance of this debate for the International society. This article aims to provide an overview of the aspects that recognize the people with disabilities as subjects of law, specifically in the opportunities for access to a regular job. The purpose is to give a perspective on the real barriers faced by this group of people when they fully participate in the development of their lives. Offering a multidisciplinary perspective, highlighting the joint work with non-governmental organizations, specifically professionals in the area, through concrete actions to eradicate discriminatory policies and generate spaces for inclusion and equality from an early age.
\end{abstract}

Key words: disability; Human Rights; inclusion; access to employment. 


\section{INTRODUCCIÓN}

En un siglo donde la igualdad, el reconocimiento de la dignidad de la persona y la no discriminación son algunos de los grandes objetivos a alcanzar, los derechos humanos se presentan como la columna vertebral donde construir una sociedad más inclusiva y equitativa.

Hoy, según la Organización Mundial de la Salud (OMS) el 15\% de la población mundial está constituida por personas con discapacidad, es decir, alrededor de 1 de cada 7 personas tiene o ha tenido algún grado de deficiencia física o mental ${ }^{1}$.

Hablar del derecho de las personas con discapacidad implica referirnos a todos los derechos fundamentales que garantizan el pleno desarrollo de una persona, desde el momento de su nacimiento hasta el fin de su vida.

Este colectivo se caracteriza por estar conformado por aquellas personas que tienen "deficiencias físicas, mentales, intelectuales o sensoriales a largo plazo que, al interactuar con diversas barreras, puedan impedir su participación plena y efectiva en la sociedad, en igualdad de condiciones con las demás".

Desde una temprana edad, aquellas personas que han nacido con alguna condición de discapacidad o que la misma les ha sobrevenido en el desarrollo de su vida, se han visto impedidas o restringidas en la participación plena en la sociedad por estar obligadas muchas veces a soluciones que tienden a su segregación. Presentándose en la mayoría de las veces como consecuencia del miedo a lo diferente o la falta de información, que genera que muchas personas no sepan cómo comportarse cuando alguien que posee condiciones diferentes se presenta ante ellos. Sobre todo, por la concepción generalizada que todas las personas con discapacidad necesitan de atención y cuidados específicos, lo que genera una tendencia a tratarlos de manera diferenciada.

Desde la institución de la familia pasando por las escuelas, las personas con discapacidad se enfrentan a barreras tanto materiales, como la imposibilidad de acceso a un edificio por falta de medios (rampas o ascensores), como sociales, al considerar que son menos capaces para realizar un trabajo por no poseer las condiciones adecuadas.

Para comprender la discapacidad como parte estructural de nuestra condición de seres humanos, debemos entender que todos y todas en algún momento de nuestras vidas, ya sea por la edad o por circunstancias excepcionales, adquiriremos algún grado de incapacidad. Es decir, la discapacidad es una circunstancia inherente al ser humano y como tal se plantea como algo más que evidente de ser trabajado y discutido.

\footnotetext{
${ }^{1}$ Dato extraído del Informe mundial sobre Discapacidad elaborado por Organización Mundial de la Salud.

${ }^{2}$ Artículo 1. Convención Internacional sobre los Derechos de las Personas con Discapacidad.
} 
Es importante remarcar los grandes avances que se han realizado en la materia, no solo desde los tecnológicos sino también en lo educativo, lo cultural y sobre todo lo social, ya que existe un marcado número de espacios de debate y de acción social que tienden a aumentar la importancia de esta situación de cara al futuro.

El punto que aquí es fundamental mencionar es el de las políticas públicas por parte de los Estados, específicamente las acciones de inclusión en los ámbitos laborales.

El reconocimiento del derecho al empleo de las personas con discapacidad encuentra su mayor inspiración en la Convención de Naciones Unidas (CPCD) del 13 de diciembre de 2006, sobre las Personas con Discapacidad, donde expresamente se hace referencia a la importancia del goce de todos los Derechos Humanos y libertades fundamentales consagrados por Naciones Unidas por este colectivo del cual diversos textos constitucionales de diferentes estados han hecho eco.

Es fundamental determinar que la inclusión no implica reconocer a todas las personas como iguales, porque en esencia no lo somos, sino considerar que aquellas que tengan condiciones similares sean respetadas de manera igual. Y aquí radica el punto central, que cuando nos referimos a los derechos de las personas con discapacidad, ello implica hablar de iguales oportunidades para aquellas personas que poseen condiciones diferentes. Consiste en plantearse que se les deben reconocer aquellos derechos que son inherentes a su persona, por el simple hecho de serlo, y que se ven consagrados de manera específica por la CPCD. Por lo tanto, hablar de discapacidad no implica hablar de derechos especiales basados en sus incapacidades, sino simplemente de derechos específicos para un sector que merece iguales oportunidades.

\section{PERSONAS CON (DIS)CAPACIDAD}

La ley, cuya fuente principal son los hechos que surgen en el devenir de la sociedad, va mutando con el tiempo fruto de una evolución en el pensamiento y en las necesidades imperantes en un momento determinado.

A lo largo de la Historia términos como "deficiencia mental" "minusvalía" "personas impedidas" o "incapacitadas" fueron algunas de las denominaciones empleadas para designar a este grupo de personas. Un número importante de textos normativos emplean estas designaciones, discriminatorias en sí mismas, creando la base sobre la que se asentaba todo el sistema de protección de los derechos de las personas con discapacidad.

El abandono de estos conceptos promovidos por la CPCD fue el principal avance en las conquistas alcanzadas por este colectivo. La discapacidad ya no 
será considerada en su vinculación con procesos de rehabilitación y condiciones de salud, sino que evoluciona hacia una concepción más social y estructural; no será apreciada como una enfermedad, sino una condición de la persona, en su reconocimiento como sujeto de derechos. No como sujetos deficientes, no como personas que sufren una enfermedad o un padecimiento, sino simplemente personas con una condición: la de la discapacidad.

A partir de este cambio en la concepción de las personas con discapacidad y al considerarlos como sujetos de derechos capaces de realizar determinados actos, fue inevitable una modificación de todo el conjunto de Ordenamientos normativos que, utilizando la terminología antigua, eran un medio más de discriminación hacia este colectivo.

Un ejemplo claro de ello es la Declaración de los Derechos de los Impedidos de 1975 de Naciones Unidas 3 que, si bien trae aparejado el tratamiento de la problemática dentro de la esfera Internacional, su texto está compuesto de un número elevado de términos que tienden a la exclusión de las personas con discapacidad al partir de la base de considerarlos "impedidos".

Así reconocerlos como sujetos de derechos, los hace también responsables de obligaciones y por tanto del cumplimiento de determinados deberes (Sánchez Ferriz, 1989; Peces-Barba Martínez, 1995). Aquí no solo interesa la conquista de los derechos sino también de todas aquellas obligaciones que deberán afrontar dentro del ejercicio de la vida civil y que contribuyen a la propia dignidad de una persona. No solo importa que se los considere en su calidad de personas iguales ante la ley sino de todo el entramado que conlleva ser legalmente capaz, porque allí es donde radica la igualdad legal ${ }^{4}$.

Si hacemos hincapié en lo ya dicho, las personas con discapacidad son vistas no como sujetos carentes de capacidades sino por el contrario, como personas capaces que poseen ciertas limitaciones.

Al quitar la etiqueta de incapacidad absoluta, el legislador reconoce a estas personas como sujetos de derechos y por lo tanto como aptos para ejercer determinados actos de la vida civil, como será en este caso, la de ocupar un empleo determinado.

Y ello es lo valeroso de todo el asunto, que un cambio en la manera de considerar a las personas puede generar, no solo una mayor inclusión social y por tanto de participación en espacios como es el empleo, sino también en la normalización de

\footnotetext{
${ }^{3}$ En su artículo 1 la Declaración establece: "1. El término "impedido" designa a toda persona incapacitada de suvenir por sí misma, en su totalidad o en parte, a las necesidades de una vida individual o social normal o a consecuencia de una deficiencia, congénita o no, de sus facultades físicas o mentales".

${ }^{4}$ El artículo 12.2 de la CPCD establece "2. Los Estados Parte reconocerán que las personas con discapacidad tienen capacidad jurídica en igualdad de condiciones con las demás en todos los aspectos de la vida"
} 
situaciones que, al abandonar su condición de tabú, pasan a conformar un aspecto más de la sociedad.

El núcleo principal de los derechos de las personas con discapacidad, y su naturaleza, lo que permitirá que emplear a una persona con discapacidad no suponga una imposición o un acto mediante el cual el empleador merezca un reconocimiento especial, consiste en la eliminación de las barreras socialmente construidas, la concepción de que las personas con discapacidad tienen necesidades especiales, y se comience a incluirlos en espacios que hasta el momento no se les da la oportunidad de participar.

La inclusión comienza por el entorno familiar, para educar a niños tolerantes en la diferencia, que luego se conviertan en jóvenes inclusivos y más tarde en adultos capaces de vivir en la diversidad.

\section{ACCESO AL EMPLEO: ¿UNA UTOPÍA O UNA NECESIDAD COLECTIVA?}

El empleo es lo que permite sentirnos sanos y activos mentalmente, no solo es una herramienta para satisfacer necesidades, sino que nos facilita ser miembros activos de la sociedad, presentándose como un espacio para desarrollar aptitudes y talentos, es decir, forma parte de la dignidad de una persona. El ámbito laboral es, sin lugar a dudas, el espacio que permite desarrollarse, integrarse socialmente, sentirse satisfecho, contar con ingresos para mantearse a sí mismo o a una familia, adquirir un producto o pagar por un determinado servicio, en definitiva, es lo que dota a una persona de independencia.

Es por tanto fundamental que cualquier ser humano pueda acceder al menos a la posibilidad de buscar un empleo que se adecue a sus necesidades, cuestión que no se permite a todas las personas con discapacidad.

Según la $\mathrm{CPCD}^{5}$ el derecho de acceso a un empleo debe hacerse en condiciones iguales que las de cualquier persona, que su elección sea libre, prohibiéndose

${ }^{5}$ Artículo 27 Convenio Internacional de los Derechos de las Personas con Discapacidad. Trabajo y empleo 1. Los Estados Parte reconocen el derecho de las personas con discapacidad a trabajar, en igualdad de condiciones con las demás; ello incluye el derecho a tener la oportunidad de ganarse la vida mediante un trabajo libremente elegido o aceptado en un mercado y un entorno laborales que sean abiertos, inclusivos y accesibles a las personas con discapacidad. Los Estados Partes salvaguardarán y promoverán el ejercicio del derecho al trabajo, incluso para las personas que adquieran una discapacidad durante el empleo, adoptando medidas pertinentes, incluida la promulgación de legislación, entre ellas: a) Prohibir la discriminación por motivos de discapacidad con respecto a todas las cuestiones relativas a cualquier forma de empleo, incluidas las condiciones de selección, contratación y empleo, la continuidad en el empleo, la promoción profesional y unas condiciones de trabajo seguras y saludables; b) Proteger los derechos de las personas con discapacidad, en igualdad de condiciones con las demás, a condiciones de trabajo justas y favorables, y en particular a igualdad de oportunidades y de remuneración por trabajo de igual valor, a 
la discriminación e incentivando la participación de manera activa en la protección de sus derechos, como así también a las posibilidades de formación y capacitación. Es decir, el ejercicio del derecho en todas sus formas de expresión. De la misma manera, los Estados se comprometen a adoptar las medidas necesarias para proteger y promover el ejercicio del derecho al trabajo, incluso para las personas que adquieran una discapacidad durante el empleo, entre estas medidas se encuentran las de promover una igualdad de oportunidades y de remuneración por trabajo de igual valor, a condiciones seguras y saludables, incluida la protección y prohibición de actitudes discriminatorias como el acoso, y a la reparación por agravios sufridos, así como en la medida de lo posible al momento de emplear personas con discapacidad prohibir que por su condición o cualquier otra circunstancia sean sometidas a esclavitud, servidumbre y que estén protegidas, en igualdad de condiciones con las demás, contra el trabajo forzoso u obligatorio.

Las limitaciones en el campo del empleo para las personas con discapacidad solo son la piedra angular del problema, pero no la única, ni la primera. El inconveniente radica en la existencia de barreras impuestas respecto a considerar que una persona con discapacidad no solo es menos eficiente, sino que no goza de las aptitudes para ejercer este derecho de manera plena.

Reforzar la educación y la formación es esencial, porque sin la primera parte de la cadena el resto de los eslabones carecen de fuerza para obligar a los Gobiernos a tomar parte activa en el reconocimiento pleno de este derecho.

El punto principal radica en que hoy el empleador mantiene la creencia de que la persona con discapacidad no es tan productiva como una persona sin discapacidad, generando desde el principio que exista un impedimento que va más allá de la propia persona y sus habilidades o no para realizar determinado trabajo. Es fundamental que para poder acceder a un empleo digno se haya otorgado

\footnotetext{
condiciones de trabajo seguras y saludables, incluida la protección contra el acoso, y a la reparación por agravios sufridos; c) Asegurar que las personas con discapacidad puedan ejercer sus derechos laborales y sindicales, en igualdad de condiciones con las demás; d) Permitir que las personas con discapacidad tengan acceso efectivo a programas generales de orientación técnica y vocacional, servicios de colocación y formación profesional y continua; e) Alentar las oportunidades de empleo y la promoción profesional de las personas con discapacidad en el mercado laboral, y apoyarlas para la búsqueda, obtención, mantenimiento del empleo y retorno al mismo; f) Promover oportunidades empresariales, de empleo por cuenta propia, de constitución de cooperativas y de inicio de empresas propias; g) Emplear a personas con discapacidad en el sector público; h) Promover el empleo de personas con discapacidad en el sector privado mediante políticas y medidas pertinentes, que pueden incluir programas de acción afirmativa, incentivos y otras medidas; i) Velar por que se realicen ajustes razonables para las personas con discapacidad en el lugar de trabajo; j) Promover la adquisición por las personas con discapacidad de experiencia laboral en el mercado de trabajo abierto; k) Promover programas de rehabilitación vocacional y profesional, mantenimiento del empleo y reincorporación al trabajo dirigidos a personas con discapacidad. 2. Los Estados Parte asegurarán que las personas con discapacidad no sean sometidas a esclavitud ni servidumbre y que estén protegidas, en igualdad de condiciones con las demás, contra el trabajo forzoso u obligatorio.
}

icade. Revista cuatrimestral de las Facultades de Derecho y Ciencias Económicas y Empresariales, $n^{2} 106$ enero-abril 2019, ISSN: 2341-0841 
previamente la posibilidad de capacitación y formación necesarios para el ejercicio de determinado puesto laboral.

Y esto resulta paradójico ya que, en cualquier solicitud de empleo lo que se buscará será gente capaz de realizar las tareas específicas del puesto, es decir, el acento estará puesto en lo que determinada empresa busca en su futuro empleado, de que contratará a la persona por lo que sabe hacer con la esperanza de que adquiera mayor experiencia a medida que se desempeña en su puesto. Entonces ¿por qué en el momento de pensar en contratar a una persona con discapacidad se piensa en lo que no puede hacer y no en aquello para lo que realmente estaría capacitada?

Sí es real y no se busca aquí idealizar las situaciones de inclusión y creer que todas las personas estamos capacitadas para realizar cualquier empleo, o que una persona con una discapacidad severa que le impida, por ejemplo, moverse o desplazarse de manera independiente pueda realizar cualquier empleo. Lo que se busca es que los empleadores o potenciales empleadores entiendan que el límite impuesto a este colectivo está en una concepción social de que la discapacidad muchas veces es sinónimo de inutilidad, cuando existen personas con un gran potencial que no se ve aprovechado.

Considerar que las personas con discapacidad son un grupo de empleados más dentro de un sistema laboral (Rojas Buendía, 2018, pp. 233-236), que gozan de una protección especial, implica aumentar mano de obra y por lo tanto capital de producción para la economía de un país. Al dotarlos de puestos de trabajo, no solo se le otorga ventajas económicas para ellos mismos, sino que es un sector de la sociedad que pasa de ser desempleado, a ser totalmente activa, que aporta en ingresos y en contribuciones, incluso si se quiere verlo desde una perspectiva económica, convirtiéndose en consumidores al mercado.

Según el Informe Mundial sobre Discapacidad de la Organización Mundial de la Salud (OMS) existen una serie de cuestiones determinantes de la discapacidad, en primer lugar que los niños con dicha condición tienen menos posibilidades de acceder a la educación; en segundo lugar que las personas con discapacidad tienen mayor probabilidades de ser desempleadas y en caso de no serlo, de recibir un salario menor del que recibiría cualquier persona por igual puesto e incluso que deben afrontar costos extras derivados de su condición, lo que afecta directamente a su economía; y por último que aquellos hogares en los que vive una persona con discapacidad tienen una mayor tendencia a experimentar inseguridad alimentaria, vivienda deficiente, falta de acceso al agua potable y saneamiento, así como también de acceso inadecuado a la salud. 
Si partimos de la premisa de que los Objetivos de Desarrollo Sostenible (ODS) ${ }^{6}$ tienen como iniciativa principal la de erradicar la pobreza, si a ello le sumamos que el número de personas desempleadas con discapacidad es mayor que de aquellas que no lo son y que la pobreza puede aumentar el riesgo de discapacidad ${ }^{7}$ entonces el nivel de empleo de este sector es una necesidad de la sociedad en su conjunto.

\section{IMPORTANCIA DE LAS ACCIONES CONCRETAS}

El cambio de perspectiva que la CPCD trajo aparejado con respecto a las acciones que deben realizar los Gobiernos, no solo inciden en el fomento de la normativa vinculada a la materia, sino en la implementación de medidas tendientes a concienciar a la sociedad para garantizar, entre otros derechos, el acceso al empleo en iguales condiciones.

Lamentablemente hoy, el número de Estados que implementan acciones en contra de las políticas discapacitantes son escasas y en muchos casos la labor realizada resulta deficiente.

Ejemplo de esto es el Tercer Plan de Acción para Personas con Discapacidad ${ }^{8}$ que arroja resultados concretos al determinar que, en una Comunidad como la de Madrid y que serviría de referencia al resto del mundo, es necesario avanzar en el establecimiento de políticas de accesibilidad de manera evidente.

Por ello ante esta falta de acción por parte de los Gobiernos, la labor por las organizaciones especializadas en inclusión y el trabajo social de entidades sin fines de lucro es algo fundamental.

\section{EXPERIENCIA EN AMÉRICA LATINA}

Existe en la ciudad de Córdoba en Argentina una Fundación llamada Gaude ${ }^{9}$ que cuenta con un Centro de Rehabilitación para Personas con Discapacidad

\footnotetext{
${ }^{6}$ La Agenda 2030 y los Objetivos de Desarrollo Sostenible establecida por Naciones Unidas en 2015 y de aplicación en 2016, contiene 17 de los Objetivos a cumplir. Basada en los Objetivos de Desarrollo del Milenio busca alcanzar medidas tendientes a erradicar la pobreza, cuidar el planeta y garantizar las libertades fundamentales para la paz y la prosperidad de la sociedad Internacional.

${ }^{7}$ Informe Mundial de la Discapacidad, 2011, p. 11.

${ }^{8}$ La Comisión Interdepartamental para la Coordinación y la Atención Integral de las personas con discapacidad aprobó el 14 de noviembre de 2016, el Informe de la Evaluación Final del Tercer Plan de Acción para Personas con Discapacidad de la Comunidad de Madrid. Este Plan ha sido el marco de referencia de las políticas de discapacidad en la Comunidad de Madrid entre 2012 y 2015.

${ }^{9}$ Fundación Gaude fue creada en 1990, está conformada por un equipo multidisciplinario que tiene el espíritu de combinar los conocimientos y la experiencia para mejorar la calidad de vida de las personas con discapacidad visual y que puedan interactuar con independencia en el hogar, la escuela, y en todos los espacios en que se desenvuelvan (http://fundaciongaude.org.ar/).
} 
Visual de la que me enorgullece ser parte. Hace un año decidimos comenzar un proyecto de capacitación de jóvenes entre 18 y 30 años, con ceguera y disminución visual, para ingresar al mercado laboral.

Llevamos a cabo un trabajo de capacitación previa, donde los jóvenes tuvieron la posibilidad de realizar simulacros de entrevistas con personal del área de Recursos Humanos de empresas privadas para poder experimentar cuales serían las situaciones con las que a futuro se podrían encontrar. De las entrevistas, lo que más pudimos aprender fue que si bien las barreras externas existen, nuestros jóvenes se auto definen a sí mismos a partir de sus propias limitaciones. A partir de ello y con un trabajo multidisciplinario de profesionales de las diversas áreas logramos que comenzaran a hablar de sus propias capacidades personales, que es aquello que podrían aportar a un empleo, que se sentían capaces de realizar con una formación previa determinadas y cuáles eran sus expectativas de desarrollo a futuro.

Así nos encontramos con que la primera barrera a derribar es la propia percepción de los jóvenes, muchas veces sostenida por vivir una vida de prejuicios, de creer que sus impedimentos eran tales que no son aptos para realizar ninguna clase de empleo.

Por otro lado nos encontramos con que un elevado porcentaje de empleadores estaban dispuestos a contratar a nuestros aprendices en una primera instancia pero que, luego nacía en ellos una serie de dudas al creer que debían dedicar más horas de las necesarias en atender a su futuro empleado o empleada, o por considerar que retrasarían la producción de su cadena de montaje o simplemente por no saber cómo tratarlos sin un profesional de por medio, lo que llevó a que de diez personas solo dos consiguieran trabajo finalmente.

Esto quiere decir, que la segunda barrera a la que se enfrentan en el momento de buscar un empleo es el miedo de las personas a tratar con alguien que creen que es diferente. Y con ello nos dimos cuenta de que el problema comenzaba desde antes y que si buscábamos conseguir posibilidades reales de empleo debíamos salir a capacitar a la gente, a derribar esos miedos y prejuicios y demostrar que una persona con discapacidad está incluso más capacitada para realizar determinadas tareas que cualquiera otra persona que no lo sea.

Con esto se quiere poner en evidencia que a día de hoy todavía deben existir instituciones, como esta fundación, que crean espacios de colocación de las personas con discapacidad en el empleo por que no existen políticas públicas concretas y eficientes que hagan creer que las barreras están para ser superadas. 


\section{CONCLUSIONES: CREAR POSIBILIDADES DESDE LO IMPOSIBLE}

Existen diversas razones por las que esta problemática debe ser colocada en un lugar de preferencia dentro de los debates sociales y, por tanto, de la proyección de medidas concretas de acción. El progresivo aumento de población con discapacidad es algo que debe traer a las diversas esferas de debate público mejores y reforzadas políticas públicas por parte de los gobiernos, pero poniendo énfasis en diversos aspectos.

1. En primer lugar, en la promoción de mayores espacios de participación social donde se dé intervención a las mismas personas con discapacidad. Ámbitos de intercambio de propuestas donde se puedan plasmar reales compromisos para palear las numeras políticas discriminatorias existentes en la sociedad.

2. Crear medidas de acción tendentes a capacitar a las pequeñas y grandes empresas. Que no sea solo a través de la intervención de los Gobiernos, sino que los empleadores vean los beneficios reales de contar con personas con discapacidad dentro de su plantilla.

3. Suscitar el acercamiento entre los niños, promoviendo medidas de inclusión, desde abordajes multidisciplinarios para lograr construir sociedades tolerantes y respetuosas de los derechos humanos.

4. Fomentar la actuación de los profesionales de la materia, quienes trabajan a través de diversas organizaciones, tanto públicas como privadas, muchas veces incluso contra las mismas acciones de los Gobiernos, para un abordaje multidisciplinar y de intervención temprana.

5. Fundamentalmente y, sobre todo, utilizar la empatía como canal de comunicación, para intentar comprender que muchos de los obstáculos a los que se enfrentan las personas con discapacidad son evitables.

Muchos son los espacios que quedan por conquistar, no solo en área de la discapacidad, sino de la inclusión en general. El trabajo es constante y aunque parezcan debates menores son circunstancias que debemos considerar de cara al futuro. Porque las imposibilidades son reales, pero las limitaciones están construidas sobre la base de prejuicios. De lo diferente es de donde nacen los nuevos desafíos, las nuevas preguntas y por tanto los nuevos derechos a conquistar. Porque la incapacidad va más allá de una limitación física, ser incapaces lo seremos todos al fin de cuentas y no por ello dejaremos de ser menos personas. 


\section{BIBILIOGRAFÍA}

Campoy Cervera, I. \& Palacios, A. (2007). Igualdad, no discriminación y discapacidad: una visión integradora de las realidades española y argentina. Madrid: Dykinson-Instituto de Derechos Humanos Bartolomé de las Casas.

Casado Pérez, D. (2004). Conocimiento y gestión del empleo de las personas con discapacidad. Revista del Ministerio del Trabajo y Asunto Sociales de España, 50, 47-71.

Dirección General de Atención a Personas con Discapacidad de la Comunidad de Madrid y Centro de Estudios Económicos Tomillo (2016). Tercer plan de acción para personas con discapacidad de la Comunidad de Madrid 2012-2015: Informe de Evaluación Final. Disponible en: http://www.madrid.org/es/transparencia/sites/default/files/plan/document/193_57_evaluacion_final_del_tercer_plan_de_accion._publicamadrid.pdf (consultado: 01/02/2019)

Ganzemuller Roig, C. \& Escudero Moratalla, J. F. (2005). Discapacidad y derecho: tratamiento jurídico y sociológico. Barcelona: Editorial Bosch.

Lorenzo, R. de (2004). El futuro de las discapacidades en el mundo: El empleo como factor determinante para la inclusión. Revista del Ministerio de Trabajo y Asuntos Sociales de España, 50, 73-89.

Organización Mundial de la Salud (OMS) (2011). Informe Mundial sobre la Discapacidad. Disponible en: https://www.who.int/disabilities/world_report/2011/ summary_es.pdf (consultado: 13/12/2018)

Organización Internacional del Trabajo (OIT) (2014). Lograr la igualdad de oportunidades en el empleo para las personas con discapacidad a través la legislación: directrices. Disponible en: https://www.ilo.org/wcmsp5/groups/public/_--ed_ emp/---ifp_skills/documents/publication/wcms_322694.pdf

Peces-Barba Martínez, G. (1995). Curso de derechos fundamentales. Teoría general, (con la colab. de Asís, R. De, Fernández Liesa, C. R. y Llamas, A.). Madrid: BOE.

Rey Pérez, J. L. \& Mateo Sanz, L. (eds.) (2018). El empleo de las personas con discapacidad: Oportunidades y desafíos. Madrid: Editorial Dykinson.

Rojas Buendía, M. M. (2018). Ajustes y apoyos universales necesarios en el ámbito laboral ordinario para la inclusión social de las personas con diversidad funcional. En Rey Pérez, J. L. \& Mateo Sanz, L. (eds.) (2018). El empleo de las personas con discapacidad: Oportunidades y desafíos (pp. 225-238). Madrid: Editorial Dykinson.

Sánchez Ferriz, R. (1989). Estudio sobre las libertades. Valencia: Tirant Lo Blanch. 